\title{
THE EFFECT OF SCREENED COULOMB INTERACTION ON THE OPTICAL PROPERTIES OF EuS/PbS/EuS FINITE CONFINING POTENTIAL QUANTUM WELL
}

\author{
K. H. AHARONYAN \\ Physics Department, State Engineering University of Armenia, \\ Yerevan 375009, Armenia \\ ahkamo@yahoo.com \\ E. M. KAZARYAN \\ Physicotechnical Department, Russian-Armenian State University, \\ Yerevan 375051, Armenia
}

\begin{abstract}
We theoretically investigate excitonic effects on the optical properties of quasi-two dimensional realistic $\mathrm{EuS} / \mathrm{PbS} / \mathrm{EuS}$ finite confining potential quantum well. The strong contrast of material parameter's across the well interfaces and the characteristic band-nonparabolicity effect of lead salt semiconductor are taken into account. In presence of medium polarization a feasibility of the screened Coulomb potential in quantum well is examined and appropriate screening radius is revealed for the first time. The screened binding energy investigated while a variational approach has been used. Depend on the realistic nanostructure specifics a monotonic decrease of the enhanced binding energy is received when in-plane carrier density/temperature ratio parameter increases. Exciton absorption spectra near the band edge within the effective-mass approximation is examined. Coulomb potential having a cutoff type is used, that efficiently represented the potential in the realistic quantum well. Screened exciton factor, which is the absorption intensity enhancement of the unbound (continuum) exciton states that are located above the band edge, is used. Plot of the dependence of the exciton factor on the exciton pair energy is given.
\end{abstract}

Keywords: Dielectric confinement; exciton; binding energy; absorption.

\section{Introduction}

Much of the interest to the quasi-two-dimensional (Q2D) electron gas (EG) optical properties in layered semiconductor structures (quantum wells $(\mathrm{QW})$, superlattices) is initiated both by fabrication advances and optoelectronic applications of these system in lasers and related technology. Optical properties of Q2D EG attract interest mainly owing to $\mathrm{Q} 2 \mathrm{D}$ excitons, which in contrast to $3 \mathrm{D}$ case exist, even at room temperature ${ }^{1}$. Carriers in a Q2D EG usually could be produced by intense optical illumination or by modulation doping and are confined in an active layer with compressed wave functions (quantum confinement (QC)). Thus, an excitonic behavior becomes enhanced due to the electron(e)-hole(h) reduced in-plane distance that leads to a larger overlap probability for the $\mathrm{e}-\mathrm{h}$ pair $^{2-4}$. The results are the increased binding energy, as well as enhanced contrast between the $1 \mathrm{~s}$ exciton peak and unbound-pair continuum, despite large density of 
thermal phonons. In particular, exciton recombination gives the main contribution to the photoluminescence spectra and even excited bound states could be observed in optical measurements ${ }^{5-6}$. In Q2D structures an advanced selective doping makes it possible to investigate a charged electron (hole) system rather than by the neutral electron-hole plasma like in the 3D samples. In contrast to the 3D system, in QW structures it is achievable coexistence of Q2D electron (hole) channel with the photoexcited excitons ${ }^{7}$. In this conditions the screening of the excitons, as consequence of Coulomb correlation between the carriers modifies the Q2D optical properties more. This essentially affects on both the stability of excitons and the character of large scale fluctuations in QW. The effect of free carrier screening is twofold. Firstly, free carriers screen the polarization field between the exciton and free carriers, thus reducing their interaction and stabilizing the exciton. Secondly, free carriers screen the exciton itself with decreasing of the binding energy of the bound state ${ }^{8-10}$ and altering the interband absorption spectra in the unbound-pair continuum ${ }^{11-14}$. The way that free carriers screen the bare Coulomb potential depends on a dimensionality of the system. In Q2D system a real -space screened potential has power law spatial dependence at large distances ${ }^{15-16}$. Thus, screening by Q2D free carriers is less effective than in the 3D, where a screened potential decays exponentially in real space. Inside of the Q2D system screening effects decrease because free carriers can only screen the field between any two charges. While a part of the field extending outside the confinement region remains unscreened.

In fact, real semiconductor QW possess both nonzero width and finite height of the potential barrier. As follows, the carriers in QW are described by wave functions that exponentially decay in barrier region but are still nonzero there. Due to the selective doping, free carrier densities could govern the strength of interaction between the particles. The latter with the parallel adjustment of the structural parameters (barrier height, QW width and strong contrast of the material parameters in heteroboundaries (MPCH )) leads to noticeable changes in the optical properties of QW. In this view the strong dielectric contrast between the QW and barrier (dielectric confinement (DC)) takes on special importance, because it can turn up the Coulomb interaction in QW strongly in relative to homogenous material ${ }^{17-18}$. In Ref. 11 the effect of Q2D screening on optical absorption for an infinite confining model has discussed when dielectric constants mismatch is strong only for just one heteroboundary, while for the other one a DC effect is negligible (an inversion channel model with a strong DC). For the semiconductors with simple spherical bands, the analytical expressions both for the Q2D screened Coulomb potential and Sommerfeld factor $(S)$ determining contribution of screened exciton effects to the optical interband absorption is obtained. Recently ${ }^{19-20}$ in realistic $\mathrm{EuS} / \mathrm{PbS} / \mathrm{EuS}$ finite confining potential QW, the screened interaction problems are discussed. The screened potential features for the two long wave limit asymptotic cases are revealed. Calculations are carried out for the logarithmical and exponential types of the potential asymptotic limits like in the case of unscreened Coulomb center ${ }^{21-22}$. In these works presence of MPCH an enhanced binding energy analytical expressions of the donor center and exciton have been obtained, respectively. 
In present work we examine screened exciton properties of realistic EuS / $\mathrm{PbS} / \mathrm{EuS}$ confining potential QW for the alternating asymptotic limit of screened potential. The latter possess more barrier influence than the logarithmical type potential because it depends on the small dielectric constant of the barrier. In this case both the screened exciton binding energy and screened excitonic Sommerfeld factor have been studied for the realistic structure under the MPCH and in presence of band nonparabolicity (NPB) effect that is specific for lead salt materials.

\section{The Potential Function}

The model used here is that of charge carriers confined to move in the rectangular QW potential along $z$ direction (for details see Ref. 20, 21) and interacting via the attractive screened Coulomb potential. A problem of screened Q2D Coulomb potential for the first time has been studied by Ritova ${ }^{15,17}$. In Ref. 17 a strong contrast between the dielectric constants of QW and barrier is accepted $\left(\varepsilon_{r}=\varepsilon_{w} / \varepsilon_{b} \gg 1\right)$ and the barrier media on each side of QW possess the same dielectric constant $\varepsilon_{b}$. For this case modified Bessel function-type potential is used

$$
V_{S}(\rho)=-\frac{2 e^{2}}{\varepsilon_{w} d} K_{0}\left(\rho / \rho_{d}\right) .
$$

Afterwards, in Ref. 11 for the case of inversion channel-like QW with particular DC $\left(\varepsilon_{w} \approx \varepsilon_{b 1}\right.$ and $\varepsilon_{w}>\varepsilon_{b 2}$ ) the 2D Debye - Hückel - type potential is obtained

$$
V_{S}(\rho)=-\frac{2 e^{2}}{\varepsilon_{w}} \frac{\exp \left(-\rho / \rho_{d}\right)}{\rho} .
$$

In Eqs. (1), (2), when one subband is filled (size quantum limit) the screening radius is equal to ${ }^{17} \rho_{d}=\sqrt{d / 2 q_{S}}$, where $2 \mathrm{D}$ screening parameter $q_{S}$ has been given by ${ }^{9}$

$$
q_{S}=2\left[1-\exp \left(-\pi \hbar^{2} n_{S} / m_{w} k_{B} T\right)\right] / a_{0} .
$$

Here $d$ is the QW width, $n_{S}$ is the $2 \mathrm{D}$ free carrier density, $a_{0}=\varepsilon_{w} \hbar^{2} / m_{w} e^{2}$ is the bulk QW material electronic Bohr radius, $k_{B} T$ is the thermal energy, $m_{w}$ is the carrier effective mass in QW semiconductor.

In Ref. 19 we have evaluated the averaged screened potential Fourier-component $<V_{S}(q)>$ in random phase approximation ( $q$ is the in-plane 2D wave vector) with DC condition. As shown, in view of the long wave $(q d<<1)$ and strong DC $\left(\mathcal{E}_{r}^{-1}<<1\right)$ conditions there are two distinct $2 \mathrm{D}$ wave vector ranges, such as $1>q d>>2 \varepsilon_{r}^{-1}$ and $1>2 \varepsilon_{r}^{-1}>>q d$, that $\left\langle V_{S}(q)>\right.$ gets the resulting analytical forms, which are:

(i) For the moderate small wave vectors with $1>q d>>2 \varepsilon_{r}^{-1}$ we have

$$
<V_{S}^{i}(q)>=-\frac{4 \pi e^{2}}{\varepsilon_{w} d} \frac{1}{q^{2}+\left(2 q_{S} / d\right)},
$$

(ii) For the small enough wave vectors with $1>>2 \varepsilon_{r}^{-1}>>q d$ we receive 


$$
<V_{S}^{i i}(q)>=-\frac{4 \pi e^{2}}{\varepsilon_{b}} \frac{1}{q+\varepsilon_{r} q_{S}} .
$$

Eq. (4) leads to the potential function from Eq. (1). The latter's asymptotic limit with the logarithmical and exponential forms are used in Refs. 19 and 20 when evaluating the binding energy of the screened donor center and exciton, respectively.

As follows from Eq. (5), for the real-space screened Coulomb potential we get

$$
V_{S}^{i i}(\rho)=-\frac{e^{2}}{\varepsilon_{b}}\left[\frac{1}{\rho}-\frac{\pi}{2} \varepsilon_{r} q_{S}\left[H_{0}\left(\varepsilon_{r} q_{S} \rho\right)-N_{0}\left(\varepsilon_{r} q_{S} \rho\right)\right]\right],
$$

where $H(x)$ and $N(x)$ are Struve and Neumann functions. In turn, screened potential $V_{S}^{i i}(\rho)$ holds if the condition

$$
\alpha_{S}=\left(q_{S} \varepsilon_{r}^{2} d / 2\right)<<1
$$

takes place among the QW material parameters due to the inequality $2 \varepsilon_{r}^{-1}>>q d$. Q2D screened potential $V_{S}^{i i}(\rho)$ for the large in-plane distances repeats the cubic powerlaw as specific to DC absent case ${ }^{16,17}$. At the same time, $V_{S}^{i i}(\rho)$, instead of a large QW dielectric constant, mainly depends from the small barrier equivalent and has been characterized by enhanced magnitude in accordance with condition $\varepsilon_{r}=\varepsilon_{w} / \varepsilon_{b} \gg 1$. As follows, in discussing case the screening parameter is defined as

$$
\rho_{S}=\left(\varepsilon_{r} q_{S}\right)^{-1}
$$

and became independent both from $d$ and $\varepsilon_{w}$ due to DC.

\section{The Binding Energy}

Let now carry out the variational calculation of the binding energy of the screened exciton with the potential $V_{S}^{i i}(\rho)$ from Eq. (5) in $\mathrm{EuS} / \mathrm{PbS} / \mathrm{EuS}$ realistic QW system. The model is solved for PbS QW grown along with [111] orientation, where the four valleys are split into one longitudinal and three equivalent oblique ones ${ }^{23}$. For $\mathrm{PbS}$ sample they are characterized by longitudinal (along [111]) and transversal (normal to [111]) band edge effective masses respectively: $m_{\ell}^{*}=0.105 m_{0}, m_{\perp}^{*}=0.08 m_{0}\left(m_{0}\right.$ is the free electron mass)). We would limit ourselves for discussing longitudinal $(\ell$ ) valley case only and used the static screening model for excitons as the approximation of more general models of dynamic screening. For bulk $\mathrm{PbS}$ sample a static dielectric constant value is $\varepsilon_{w}=170$ with exciton Bohr radius $a_{0}^{e x} \approx 205 \mathrm{~nm}$, while in bulk EuS (as a wide gap barrier material) the static dielectric constant value is $\varepsilon_{b}=10$ (Ref. 24). In europium salts, $a_{0}^{e x}$ of the order of the lattice parameter and an effective mass $m_{b} \sim m_{0}$ (Ref. 25). For the ground bound state level we choose normalized one variational parameter $\lambda$ trial 
wave function in the form $\psi(\rho)=\sqrt{2 / \pi}\left(\lambda e^{-\lambda \rho}\right)$. For the binding energy, after necessarily variational actions we get

$$
E(\lambda)=\frac{\hbar^{2} \lambda^{2} m_{r}}{2 m_{\perp}^{e x}}-\frac{4 e^{2} \lambda^{2}}{\varepsilon_{b} \Lambda}\left\{2 \lambda+\varepsilon_{r} q_{S}+\frac{2 \varepsilon_{r} q_{S} \lambda}{\Lambda^{1 / 2}} \ln \left[\frac{\varepsilon_{r} q_{S}}{2 \lambda} \frac{\sqrt{\Lambda}-\varepsilon_{r} q_{S}}{\sqrt{\Lambda}+2 \lambda}\right]\right\},
$$

where $m_{\perp}^{e x}$ is the exciton reduced mass in QW semiconductor, $\Lambda=4 \lambda^{2}+\varepsilon_{r}^{2} q_{S}^{2}, m_{r}$ is the renormalization term of the effective mass due to finite confinement. To account for the influence of NPB in lead salts, we have used an energy-dependent effective mass approach ${ }^{22,23}$, for that the expression $m_{\ell(\perp)}=m_{\ell(\perp)}^{*} n_{e(h) l}$ is used, where $n_{e(h) l}$ is the NPB term. The latter together with one-particle size-quantized parameters have been calculated in the Refs. 21, 22 and the subsequent results are used here for the discussing system. The binding energy might be obtained after minimization of Eq. (9) with regard to $\lambda$. The results are shown numerically in Fig. 1 where the binding energy is displayed as the function of the density and temperature parameters ratio $n_{S} / T$ for QW width value $d=3 \mathrm{~nm}$. The allowed interval for the parameter $n_{S} / T$ is defined in accordance with Eq. (7) and, as shown in Fig. 1, corresponds to the values of $\sim\left(10^{6} \div 10^{8}\right) \mathrm{cm}^{-2} /{ }^{0} \mathrm{~K}$. The latter matches mainly with the nondegenerate in-plane density values. As follows from the graph, the binding energy, as expected, is strongly enhanced and decreases with increase of parameter $n_{S} / T$. This distinguish enhancement takes place, at first, due to the increased influence of barrier media in discussed EuS/PbS/EuS realistic QW and, second, because screening properties are wholly suppressed for the relatively low values of the parameter $n_{S} / T$.

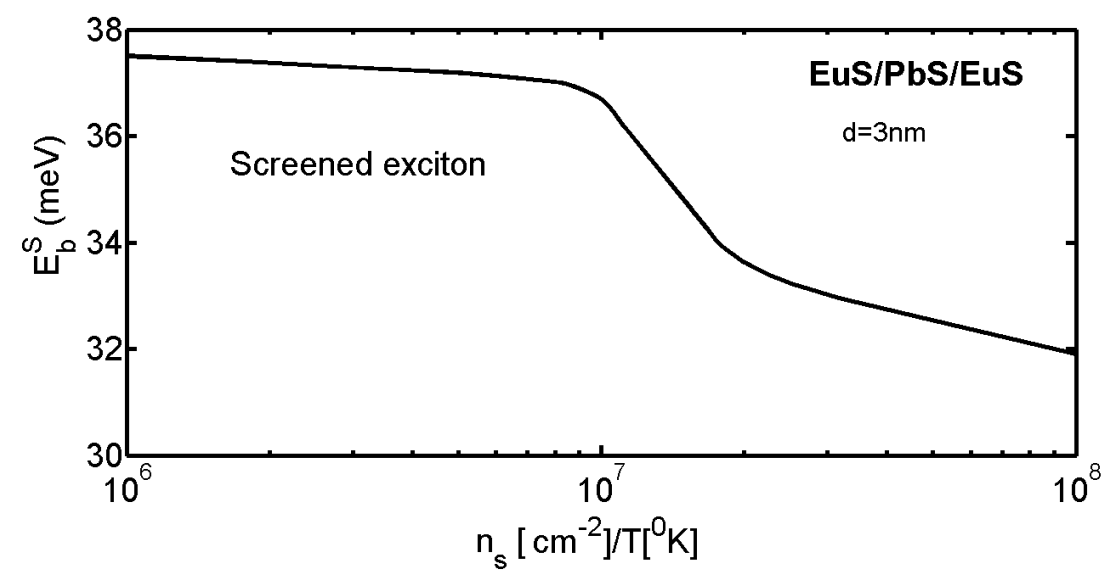

Fig. 1. The binding energy of the screened exciton as a function of the parameter ratio $n_{s} / T$. The curve corresponds to the QW width $3 \mathrm{~nm}$. 


\section{The Screened Excitonic Factor}

The absorption coefficient in a semiconductor in presence of the exciton effects is proportional to the enhanced probability which rises from residual $\mathrm{e}-\mathrm{h}$ pair correlation in the same unit cell and within the effective mass approximation this probability is given by Sommerfeld excitonic factor $S$. Here we are interested in screened excitonic absorption in the QW continuum where the pair correlation produces an almost flat spectrum in the $3 \mathrm{D}$. While in the $2 \mathrm{D}$ pair correlation gives rise from the band - to - band step value far above the gap to a value twice as large at the fundamental edge $\mathrm{e}^{1-3}$. The factor $S$ depends on the form of interaction potential, which in discussing case is described with potential function from Eq. (6). The latter cannot be solved exactly and here a cut - off Coulomb potential method is used already elaborated in the $3 \mathrm{D}^{26}$ and in the $2 \mathrm{D}^{11}$ cases, respectively. In accordance with this method the potential from Eq. (6) takes the form

$$
V_{S}(\rho)=\left\{\begin{array}{lc}
-e^{2} / \varepsilon_{b} \rho & \rho \leq \rho_{0} \\
0 & \rho>\rho_{0}
\end{array}\right.
$$

where $\rho_{0}=\max \left\{\rho_{S}, \rho_{a v}\right\}, \rho_{S}=\left(\varepsilon_{r} q_{S}\right)^{-1}, \rho_{a v}$ is the average distance between the free carriers. Note, that Eq. (10) is specified from the asymptotic behavior of the Struve and Neumann functions. As obtained in Ref. 11 for the states of zero angular momentum (only these states contribute to the absorption ${ }^{1,2}$ ) the excitonic factor $S$ in case of screened potential after Eq. (10) has the complex form

$$
S=\left|\frac{J_{0}(\chi \delta) N_{1}(\chi \delta)-J_{1}(\chi \delta) N_{0}(\chi \delta)}{\left[2 F_{1}^{\prime}-F_{1}\right]\left[J_{0}(\chi \delta \lambda)+i N_{0}(\chi \delta)\right]+F_{1}\left[N_{1}(\chi \delta)-i J_{1}(\chi \delta)\right]}\right|^{2} .
$$

In (11) $J_{n}(x), N_{n}(x), F_{1}(x)$ are the Bessel, Neumann, confluent hyper geometrical functions, respectively, $F_{1}^{\prime}$ is the derivative of the $F_{1}(x), x=\chi \delta, \chi=q a_{0}^{e x} / \varepsilon_{r}$ is the reduced energy parameter, $\delta=\rho_{0} \varepsilon_{r} / a_{0}^{e x}$ is the reduced screening parameter.

To reveal the features of excitonic absorption in realistic EuS/PbS/EuS QW the excitonic factor from Eq. (11) is analyzed both analytically and numerically. Calculations have done, as in previous section, for the material parameter restrictions related to Eqs. (5) - (8). Therefore an allowed interval for the parameter $n_{S} / T$ corresponds to the values of $\sim\left(10^{6} \div 10^{8}\right) \mathrm{cm}^{-2} /{ }^{0} \mathrm{~K}$ and the permitted in-plane $q$ vectors satisfy to the condition $q<<2 / \mathcal{E}_{r} d$, which is true for enough thin QW. As follows, for the discussed realistic structure the reduced energy parameter takes the values $\chi<<1$ and reduced screened parameter satisfies to the condition $\delta>>1$. For that case Eq. (11) reduces to the asymptotic unscreened modified form ${ }^{11}$

$$
S=\frac{\exp (\pi / \chi)}{\cosh (\pi / \chi)}\left\{1+\frac{1}{\chi \delta}\left[\frac{1}{4}+\frac{\cos (\Delta)}{\chi^{2}}\right]\right\}
$$

where $\Delta=2 \chi \delta+2 \ln (2 \chi \delta) / \chi+2 \arg [\Gamma(1-(i / \chi))]$. 


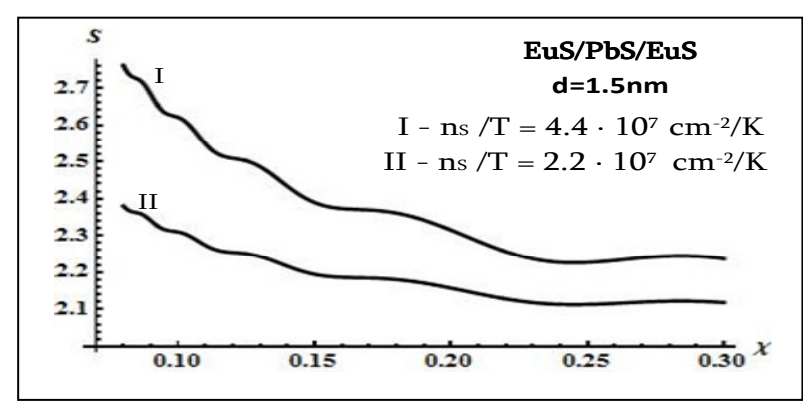

Fig. 2. The screened exciton $S$ factor as a function of the parameter $\lambda$ at fixed parameter $\chi$. The top (low) curve corresponds to the parameter value $n_{S} / T=4.4 .10^{7} \mathrm{~cm}^{-2} /{ }^{0} \mathrm{~K}$ $\left(2.2 .10^{7} \mathrm{~cm}^{-2} /{ }^{0} \mathrm{~K}\right)$ with QW width $1.5 \mathrm{~nm}$.

In Fig. 2 we plot the excitonic factor with Eq. (12) as a function of the reduced energy parameter $\chi$ at the fixed reduced screening parameter $\delta$ by taking into account realistic EuS/PbS/EuS QW material parameter restrictions $\chi<1, \delta \gg 1$. The graphical curves are displayed for the $\mathrm{QW}$ width $d=1.5 \mathrm{~nm}$ and for the fixed density / temperature ratio parameter values $n_{S} / T=4.4 .10^{7} \mathrm{~cm}^{-2} /{ }^{0} \mathrm{~K}$ ( the top curve ) and $2.2 .10^{7} \mathrm{~cm}^{-2}$ $\rho^{0} \mathrm{~K}$ ( the lower curve ), that correspond to the reduced screening parameter values $\delta=$ 16.32 and 32.65 , respectively. Received shallow oscillating absorption intensity strongly depends on the energy parameter $\chi$ and density/temperature ratio parameter $n_{S} / T$. In particular, with the value of $\chi=0.1$ an absorption intensity of the top curve $\left(n_{S} / T=\right.$ $4.4 .10^{7} \mathrm{~cm}^{-2} / 0 \mathrm{~K}$ ) exceeds pure unscreened limit $S=2$ more than $30 \%$. In turn, the intensity decreases nearly $14 \%$ with decreasing of by twice of $n_{S} / T$ parameter. With decreasing of $n_{S} / T$ and increasing of $\chi$ spectral curves tend to pure unscreened limit with $S \rightarrow 2$. As follows, the graphical data indicate to the importance of exciton effects in formation of absorption curve in EuS/PbS/EuS QW.

\section{Conclusions}

The theoretical study of screened Coulomb interaction on the exciton binding energy and excitonic absorption in realistic EuS / $\mathrm{PbS} / \mathrm{EuS}$ finite confining potential QW is carried out in presence of QW and barrier material parameter's strong contrast across the heteroboundaries. The characteristic band-nonparabolicity effect of lead salt semiconductor material is taken into account. In presence of inhomogeneous dielectric polarization of QW system an enhanced 2D Coulomb-type (Eq. (6)) screened Q2D potential and appropriate screening radius (Eq. (8)) are elaborated for the first time. A screened binding energy is investigated variationally. Depending on realistic QW specifics, the numerical analysis is provided. A very strong enhancement of the binding energy $E_{b}^{S}(d)$ in relation to tiny unscreened bulk value $(\sim 0.1 \mathrm{meW})$ is received for small QW width values and monotonic decrease of the screened binding energy is revealed with increasing of parameter $n_{S} / T$. An absorption intensity properties of the screened exciton continuum states for the realistic QW are discussed and the appropriate 
form of Sommerfeld excitonic factor is calculated numerically. The results indicate to the favorable conditions for detection of the screened exciton bound states and exciton absorption in $\mathrm{EuS} / \mathrm{PbS} / \mathrm{EuS}$ finite confining potential QW system.

\section{References}

1. S. Scmitt-Rink, D.S. Chemla and R.C. Miller, Adv. Phys. 38, 89 (1989).

2. M.Bugajski and K. Reginski, Opto-Electronic Rev. 4, 83 (1996).

3. M. Shinada and S. Sugano, J.Phys. Soc. Jpn. 21, 1936 (1966).

4. E.M. Kazaryan and R.L. Enfiadjyan, Sov. Phys. Semicond. 5, 2002 (1971).

5. E. S. Koteles and J. Y. Chi, Phys. Rev. B 37, 6332 (1988).

6. M. Gurioly, J. Martinez-Pastor and M. Collochi, Phys. Rev. B 47, 15755 (1993).

7. S. I. Gubarev, I. V. Kukushkin, S .V. Tovstonog, M. Yu. Akimov, J. Smet, K. von Klitzing and W. Wegschelder, JETP Lett. 72, 324 (2000).

8. D. A. Kleinman, Phys. Rev. B 32, 3766 (1985).

9. J. Lee, H. N. Spector and P.Melman, J. Appl. Phys. 58, 1893 (1985).

10. D. A. Dahl, Phys. Rev. B 37, 6882 (1988).

11. K. H. Aharonyan and E. M. Kazaryan, Thin Solid Films 105, 149 (1983).

12. Y. Jiang, M. C. Teich and W. I. Wang, J. Appl. Phys. 71, 769 (1992).

13. G. E. W. Bauer, Phys. Rev .B 45, 9153 (1992).

14. D. V. Kulakovski1 , S. I. Gubarev and Yu. E. Lozovik, JETP 94, 785 (2002).

15. N. S. Rytova, Sov.Phys. Doklady 10, 754 (1965).

16. F. Stern and W. E. Howard, Phys. Rev. 163, 816 (1967).

17. N. S. Rytova, Vestnik Moscow Univ. 3, 30 (1967).

18. L. V. Keldish, JETP Lett. 29, 658 (1979).

19. K. H. Aharonyan, Journal of Physics: Conference Series 350, 012015 (2012).

20. K. H. Aharonyan and E. M. Kazaryan, Physica E 44, 1924 (2012).

21. K. H. Aharonyan Physica E 43, 111 (2010).

22. K. H. Aharonyan, Physica E 43, 1618 (2011).

23. E. A. de Andrada e Silva, Phys. Rev. B 60, 8859 (1999).

24. I. K. Kikoin (ed.), Tables of physical quantities, djvu, russ. (Atomizdat, Moscow, 1976), p. 378.

25. S. von Molnar, IBM J. Res. Develop. 269, (1970).

26. G. L. Bir, G. E. Picus and A. S. Skal, Sov. Phys. Semicond. 8, 1096 (1974). 